\title{
Faire de l'histoire en Allemagne. Un guide pour les jeunes chercheurs français
}

\section{Falk Bretschneider et Mareike König}

\section{(2) OpenEdition}

\section{Journals}

Édition électronique

URL : http://journals.openedition.org/ifha/159

DOI : 10.4000/ifha.159

ISSN : 2198-8943

\section{Éditeur}

IFRA - Institut franco-allemand (sciences historiques et sociales)

\section{Édition imprimée}

Date de publication : 6 février 2011

ISSN : 2190-0078

\section{Référence électronique}

Falk Bretschneider et Mareike König, « Faire de l'histoire en Allemagne. Un guide pour les jeunes chercheurs français », Revue de l'IFHA [En ligne], 3 | 2011, mis en ligne le 19 avril 2012, consulté le 03 mai 2019. URL : http://journals.openedition.org/ifha/159 ; DOI : 10.4000/ifha.159

Ce document a été généré automatiquement le 3 mai 2019.

(CIFHA 


\title{
Faire de l'histoire en Allemagne. Un guide pour les jeunes chercheurs français
}

\author{
Falk Bretschneider et Mareike König
}

\section{NOTE DE L'ÉDITEUR}

Falk Bretschneider est maître de conférences à l'École des hautes études en sciences sociales (Paris) ; Mareike König est chargée de recherche et directrice de la bibliothèque de l'Institut historique allemand de Paris. Le présent texte reprend en grande partie les passages de l'introduction dont les deux auteurs ont fait précéder le guide qu'ils ont dirigé.

À l'heure actuelle, séjourner plus ou moins longuement à l'étranger est devenu un passage obligé pour une majorité d'étudiants et de doctorants. En principe, la science constitue un monde sans frontières par nature; depuis quelques années pourtant, les exigences en matière d'expériences internationales - notamment envers les jeunes chercheurs - se sont encore accrues. Aujourd'hui, non seulement la production du savoir a lieu dans un horizon mondial d'échanges, mais les structures mêmes de cette production dépassent de plus en plus les cadres des systèmes d'éducation nationaux avec, par exemple, l'européanisation des programmes de financement de la recherche ou l'impact - certes contestable mais néanmoins grandissant - des classements académiques des universités à l'échelle planétaire (comme le fameux classement de Shanghai). Par conséquent, avoir un profil « international » est devenu, pour la plupart des universités, un impératif qui influence également les pratiques de recrutement et les principes de formation des jeunes chercheurs.

Face à ces mutations du monde universitaire, choisir de faire une partie de ses études ou de sa thèse à l'étranger représente sans aucun doute une option attrayante. Même si celle-ci reste statistiquement toujours minoritaire, elle concerne une quantité croissante 
d'étudiants et de doctorants. Et pour cause : commencer ou continuer son activité de jeune chercheur dans un pays autre que celui dans lequel on a bénéficié de sa formation initiale n'est plus aujourd'hui comparable à une expédition en pleine jungle. Désormais, de nombreux dispositifs existent afin d'encadrer les formations, que ce soit au niveau des études de deuxième cycle ou à celui du doctorat (les cursus intégrés et la cotutelle sont certainement les plus connus d'entre eux).

\section{Un guide d'expérience(s)}

Pour autant, passer la frontière pour s'intégrer dans un autre système universitaire constitue toujours une petite aventure. Ceci est vrai également pour un passage vers l'Allemagne, pays avec lequel les échanges universitaires français s'enracinent cependant aujourd'hui dans une riche histoire longue de plusieurs décennies. En dépit des multiples contacts et des connaissances qui en résultent, traverser le Rhin pour faire sa thèse ou ses études peut toujours être le point de départ d'un parcours du combattant institutionnel, car les possibilités de bourses, de cotutelle, de cursus intégrés sont certes très nombreuses, mais dessinent un paysage labyrinthique. C'est enfin une aventure intellectuelle : les traditions scientifiques des deux pays, dans de nombreuses disciplines (et tout particulièrement en histoire), conservent des singularités et des généalogies spécifiques, que chaque chercheur (jeune ou confirmé), sans abandonner le bagage qui lui a été donné dans son pays d'origine, doit s'approprier.

Mais traverser le Rhin est aussi plus que tout cela : c'est aller d'un système universitaire vers un autre et faire l'expérience d'une réalité sociologique - on osera dire aussi : anthropologique - qui traverse les ossatures institutionnelles, qui nourrit et se nourrit des différentes traditions intellectuelles, qui s'exprime dans des domaines aussi variés que les grands rites universitaires, les rythmes de l'année ou de la carrière, le style de l'écriture académique, la fonction et l'aspect des notes de bas de pages - ou même la manière de poser les premières questions dans un entretien scientifique.

Évidemment, si le passage vers l'Allemagne reste une expérience humaine incomparable, de celles qui marquent une vie, il y a plusieurs manières de le vivre. On pourra l'envisager par exemple comme l'aventure d'un explorateur solitaire, à la recherche de sensations authentiques et éprouvées - cela va de soi et participe au charme de ce genre d'expéditions - au cours d'un itinéraire semé d'embûches. Ou, à l'inverse, on pourra le concevoir comme un voyage organisé qui, au prix d'une expérience aseptisée et dépourvue de tout contact avec la " vraie » vie des gens sur place, apporte le confort du all inclusive et la sécurité de ne passer à côté d'aucune curiosité dont l'importance n'aurait été certifiée par au moins un million de photographies floues. Enfin, on pourra l'imaginer d'une troisième façon: de celle qui essaie de préparer au mieux le voyageur, en lui fournissant au préalable les informations nécessaires, afin qu'il puisse lui-même affronter l'étrangeté dans toutes ses facettes, palpitantes, fascinantes mais aussi déroutantes. C'est cette troisième option qui marque la philosophie du guide Faire de l'histoire en Allemagne, disponible depuis quelques mois sur Internet et présenté, sous l'égide de l'Institut historique allemand de Paris, du Centre de recherches interdisciplinaires sur l'Allemagne (UMR 8131, CNRS-EHESS) et de la plateforme internationale de publication Perspectivia.net ${ }^{1}$, par une quinzaine d'auteurs français et allemands.

Des guides, il en existe toutefois de formes diverses et variées. Chacune présente ses avantages et ses inconvénients, et chacune est le résultat de plusieurs choix : ainsi ce 
guide à destination des jeunes chercheurs qui tentent l'aventure - faire de l'histoire en Allemagne - n'est-il ni un répertoire du paysage institutionnel, ni un inventaire des moyens de mener à bien un travail de recherche dans l'autre pays. Bien qu'il indique, au fil des articles, de nombreuses institutions de recherche ou d'innombrables instruments de travail, un aperçu des filières binationales ou multinationales encadrant la jeune recherche et des indications sur les partenaires les plus efficaces, il n'a pas pour ambition d'être un catalogue - et encore moins un catalogue complet - de toutes les possibilités offertes aux étudiants et doctorants pour, par exemple, obtenir des financements, réaliser des séjours ou bénéficier d'encadrements. Il ne se fixe pas non plus pour objectif de brosser le portrait de deux historiographies et de leurs interactions. Autrement dit, il n'offre pas au lecteur une histoire franco-allemande des manières de faire de l'histoire. Enfin, même s'il ne cesse d'articuler les traditions intellectuelles avec les conditions concrètes du travail scientifique, il ne se présente pas sous la forme d'un inventaire des sous-disciplines et des champs de recherche des deux pays.

En fait, l'ambition de ce guide est toute autre. Il aspire à se situer en amont de ces indications institutionnelles et de ces réflexions historiographiques, en les intégrant dans une présentation non seulement des systèmes qui organisent la recherche de part et d'autre du Rhin, mais aussi des habitus différents qui sont à la fois le résultat et le moteur quotidien de ces systèmes. Il s'intéresse donc à la différence des espaces, telle qu'elle s'exprime dans des domaines bien plus nombreux que le système universitaire - par exemple dans l'organisation de la recherche et les réflexes des chercheurs : il réfléchit à la valeur des diplômes et des concours, si différente au-delà et en deçà du Rhin; il cherche à comprendre comment se sont structurés les paysages disciplinaires, mais aussi les paysages archivistiques, les galaxies des revues ou les champs à la pointe de la recherche dans chacun des deux pays; il tente enfin d'aller jusqu'au quotidien de la recherche, de fournir des instruments pour décoder les réactions, les attentes, les nondits sur lesquels reposent (plus souvent qu'une analyse purement intellectuelle des relations scientifiques le laisserait apparaître) bien des incompréhensions ouheureusement - bien des séductions, et d'où surgissent aussi des dépaysements inattendus, au détour d'une phrase, d'un colloque ou d'un répertoire d'archives.

La logique de ce guide, au-delà de sa grande diversité thématique et de la mine de renseignements qu'il propose, est donc d'être un guide d'expérience(s). Il est fondé sur l'expérience des auteurs, familiers de ces parcours croisés entre la France et l'Allemagne et qui ont, pour nombre d'entre eux, participé à des programmes de formation (notamment doctorale) franco-allemande, par exemple dans le cadre du Centre interdisciplinaire d'études et de recherches sur l'Allemagne (CIERA), de l'Institut historique allemand de Paris (IHA) ou de la Mission historique française en Allemagne (MHFA, aujourd'hui Institut français d'histoire en Allemagne, IFHA). Ce guide ambitionne de fournir à son tour des clefs pour l'expérience totale - et pas seulement intellectuelle qu'est la recherche dans et/ou sur un autre pays. Il est fondé sur la conviction que travailler ailleurs est toujours l'occasion d'un retour réflexif sur la manière de travailler chez soi, et que le "décentrage » apporté par l'étrangeté du regard, celui que l'on pose sur les autres et que les autres posent sur soi, autorise à sortir des certitudes acquises et des automatismes par lesquels un système social - celui de la recherche comme les autres - produit et se reproduit. 


\section{Un outil pratique}

Concrètement, le guide s'organise autour de trois grands volets thématiques dont les articles, cela va de soi, sont liés entre eux par de nombreux renvois et traits d'union: après un bilan des tendances actuelles de l'historiographie outre-Rhin (Étienne François), le guide propose un regard sur les structures institutionnelles de la recherche historique allemande (Falk Bretschneider, Bernd Klesmann, Rahul Markovits), sur l'organisation de l'enseignement supérieur et les logiques sociales qui y régissent les études (Gabriele Lingelbach) et la thèse de doctorat en histoire (y compris celle préparée en cotutelle) (Falk Bretschneider et Christophe Duhamelle).

Ensuite, le guide initie le lecteur aux particularités de quelques cadres institutionnels où il trouve les ressources qui sont indispensables à son travail: les archives (Falk Bretschneider et Juliette Guilbaud), les revues d'histoire (Christophe Duhamelle), les bibliothèques (Mareike König) et le monde des ressources électroniques accessibles sur Internet (Mareike König et Annette Schläfer). Là aussi, il s'agit d'appliquer, autant que faire se peut, un "regard ethnologique " pour essayer de comprendre ce qui pourrait paraître, au premier abord, «bizarre», «étrange » ou tout simplement incompréhensible.

$\mathrm{Si}$, dans tous ces textes, l'aspect pratique des renseignements fournis est fondamental, il l'est encore davantage dans un dernier groupe de contributions qui cherchent à donner des conseils sur des questions qui peuvent paraître banales, mais se révèlent bien souvent décisives pour la réussite d'un travail de recherche à l'étranger : comment préparer son séjour (Patrick Farges) et comment le financer (Peggy Rolland et Claire Vital), comment écrire sa thèse (Elisabeth Venohr et Sébastien Rival) et comment «bien » la publier dans un contexte binational (Claudie Paye), enfin comment trouver sa place sur un marché franco-allemand du travail qui, pour des jeunes chercheurs fraîchement diplômés, offre de multiples occasions aussi bien à l'intérieur qu'à l'extérieur du monde universitaire et de la recherche (Elissa Mailänder)?

Dans tous ces articles, les auteurs ont déployé leurs efforts pour faire part de leurs expériences et pour les inscrire dans une réflexion commune sur les pratiques de la recherche dans un cadre franco-allemand. S'intégrant dans la nouvelle collection « Scholar Guides » (dont il constitue le premier volume) de la plateforme Perspectivia.net, ce nouveau guide présente enfin, par sa forme électronique, un avantage essentiel par rapport aux quelques rares entreprises semblables et parues sous forme papier: outre une très large visibilité, la publication en ligne offre la possibilité de mettre à jour les articles individuellement, ce qui semble indispensable notamment en raison des nombreux liens (vers des pages web) cités dans les textes. 


\section{BIBLIOGRAPHIE}

Internet : http://www.perspectivia.net/content/publikationen/scholar-guide/histoire-enallemagne

\section{NOTES}

1. http://www.perspectivia.net. 